\title{
ESA Atmospheric LIDAR (ATLID) testing, qualification, and calibration
}

G. de Villele, J. Pereira do Carmo, K. Wallace, B. Corselle, T. Belhadj, et al.

G. de Villele, J. Pereira do Carmo, K. Wallace, B. Corselle, T. Belhadj, P. Bravetti, A. Lefebvre, F. Chassat, T. Kanitz, K. Ghose, C. Haas, "ESA Atmospheric LIDAR (ATLID) testing, qualification, and calibration," Proc. SPIE 11852, International Conference on Space Optics - ICSO 2020, 118521J (11 June 2021); doi: 10.1117/12.2599245

SPIE Event: International Conference on Space Optics - ICSO 2021, 2021, Online Only 


\section{International Conference on Space Optics-ICSO 2020}

Virtual Conference

30 March-2 April 2021

Edited by Bruno Cugny, Zoran Sodnik, and Nikos Karafolas
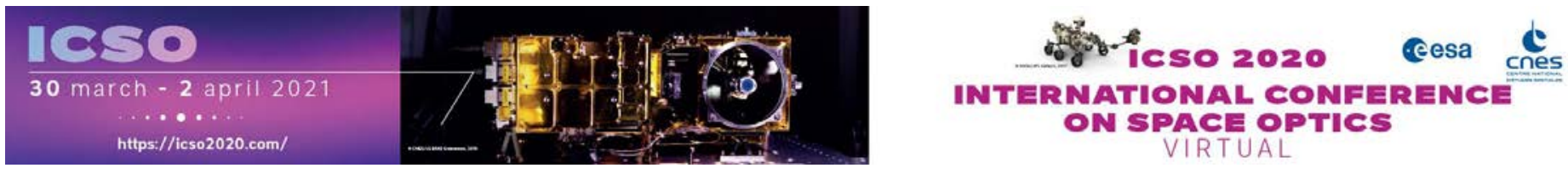

\section{ESA Atmospheric LIDAR (ATLID) testing, qualification, and calibration}

\section{Cesa isoporecestings denes}




\title{
ESA Atmospheric LIDAR (ATLID) Testing, Qualification and Calibration
}

\author{
de Villele G. ${ }^{1}{ }^{*}$, Pereira do Carmo J. ${ }^{2}$, Wallace K. ${ }^{2}$, Corselle B. ${ }^{1}$, Belhadj T. ${ }^{1}$, Bravetti P. ${ }^{1}$, Lefebvre \\ A. $^{2}$, Chassat F. ${ }^{2}$, Kanitz T. ${ }^{2}$, Ghose K. ${ }^{2}$, Haas C. ${ }^{3}$ \\ ${ }^{1}$ Airbus Defence And Space S.A.S, Toulouse, France \\ ${ }^{2}$ European Space Agency, ESA-ESTEC, Keplerlaan 1, 2202 AG Noordwijk, The Netherlands \\ ${ }^{3}$ Airbus Defence And Space GmbH, Immenstaad, Germany \\ *geraud.devillele@airbus.com
}

\begin{abstract}
The global representation of aerosol, clouds, and their impact on the energy budget remains a major source of uncertainties in global models for climate predictions, but also strong weather events in numerical weather prediction. Earth Cloud, Aerosol and Radiation Explorer (EarthCARE) mission, the sixth Earth Explorer Mission of the European Space Agency (ESA) Living Planet Programme, shall improve the understanding by three dimensional observations of global profiles of cloud, aerosol, precipitation and associated radiative properties. A major contribution will be provided by the ATmospheric LIDAR (Light Detection and Ranging), ATLID, being developed and tested by Airbus Defence and Space S.A.S in Toulouse. ATLID is a High Spectral Resolution-Polarization LIDAR and will provide vertical profiles of optically thin cloud and aerosol layers, the aerosol type, as well as the altitude of cloud boundaries. After the development, qualification and delivery of all units, ATLID integration has finally been completed and the self-standing instrument was successfully tested to demonstrate its performance under ambient and space environment conditions, in Toulouse and CSL/Liège respectively. All preliminary test results obtained up to now are very promising and indicate ATLID's capability and compliance to all performance goals and specifications. The instrument has been now delivered, to Airbus Defence and Space GmbH Immenstaad, and integrated into the EarthCARE platform. Results of ATLID ambient test campaign, EMC, mechanical test campaign, and thermal balance test campaign, along with final performance and calibration test results shall be presented in this paper.
\end{abstract}

Keywords: Lidar, backscattering, Rayleigh, Mie, laser, atmosphere, aerosols, thin clouds, remote sensing

\section{INTRODUCTION}

\subsection{EarthCARE mission and ATLID instrument}

The ATLID instrument is part of EarthCARE satellite, which stands for Earth Cloud, Aerosol and Radiation Explorer. This Satellite is a joint collaborative mission between ESA (European Space Agency) and JAXA (National Space Development Agency of Japan). Refer to [1][2][3][4][5]

EarthCARE (EC) will allow scientists to study the interactions between clouds, aerosols and radiation and relationship with Earth radiative balance at accuracy levels that will significantly improve our understanding of the highly variable associated parameters and improve predictions about the weather and future climate[6][14]. Specifically, EarthCARE scientific objectives are:

- Observation of the vertical profiles of natural and anthropogenic aerosols on a global scale, their radiative properties and interaction with clouds;

- Observation of the vertical distributions of atmospheric liquid water and ice on a global scale, their transport by clouds and their radiative impact;

- Observation of cloud distribution ('cloud overlap'), cloud precipitation interactions and the characteristics of vertical motions within clouds; 
- Retrieval of profiles of atmospheric radiative heating and cooling through the combination of the retrieved aerosol and cloud properties.

The EC payload is composed of 4 instruments: a Cloud Profiling Radar (CPR) furnished by JAXA, providing vertical profiles of clouds and evaluation of vertical speed in clouds thanks to Doppler measurementss at 94GHz [13]; a Mutli Spectral Imager (MSI) with 7 channels from $0.7 \mu \mathrm{m}$ to $12 \mu \mathrm{m}$ wavelength, used to provide contextual information about cloud type, structure and texture and some aerosols data; a Broad Band Radiometer (BBR) measuring top of atmosphere radiance [12]; an ATmospheric UV LIDAR (ATLID) providing profiles of aerosol layers and optically thin clouds.

The four instruments will provide simultaneously the vertical profiles of clouds and aerosol characteristics, together with vertical temperature and humidity profiles and the top of atmosphere radiance. The co-registered aspect of the payload data is an important aspect in allowing the measurements to be used synergistically to allow the mission objectives to be met.

The ATLID instrument is a UV LIDAR with High Spectral Resolution capability. The LIDAR emitted ultraviolet (UV) light $(355 \mathrm{~nm})$ enhances Rayleigh molecular backscattering signal; this helps to distinguish between the Mie particle aerosols with monochromatic backscattering and the molecular Rayleigh backscattering which is spectrally broadened by a few GHz. This distinction allows quantifying the extinction-to-backscatter ratio. The spectral separation is performed via the High Spectral Resolution Etalon (HSRE) included in the telescope focal plane of the instrument. A polarizer optic allows to also quantify the cross polarized backscattered signal, mainly due to certain aerosol type with oriented shapes.

The double capability of ATLID with its 3 channels (Co-polarized Mie, Co-polarized Rayleigh, Cross-polarized) leads to unique and unprecedented LIDAR products. Previous LIDAR in orbit have not yet combined the HSRE capability with the polarization backscattering measurement. The CALIPSO is a LIDAR mission with double wavelength (1064nm and 532nm), but with no HSRL capability, and the AEOLUS mission provides indirect backscattering products with Mie and Rayleigh separation but only with one single polarization.

The EarthCARE satellite will be placed in a sun-synchronous orbit, with a repeat cycle of 25 days, and a low average altitude of $408 \mathrm{~km}$. The quasi-polar orbit allows covering latitudes range between $+/-83^{\circ}$.

The ATLID LIDAR data consists of backscattered signals measured with vertical resolution of $100 \mathrm{~m}$ ( 0 to $20 \mathrm{~km}$ altitude) or $500 \mathrm{~m}$ ( 20 to $40 \mathrm{~km}$ altitude); Figure 1 . The UV laser emits pulses at $35 \mathrm{~mJ}$, with a pulse repetition frequency (PRF) of $51 \mathrm{~Hz}$, within a typical $36 \mu \mathrm{rad}$ total emission cone angle. In baseline settings, the backscattered signal is accumulated on an MCCD after two laser shots, leading to a ground sampling distance of $285 \mathrm{~m}$ from two typical $14 \mathrm{~m}$ diameter laser footprints. The main performance requirements are assessed with on ground averaging over $10 \mathrm{~km}$ of these accumulations.

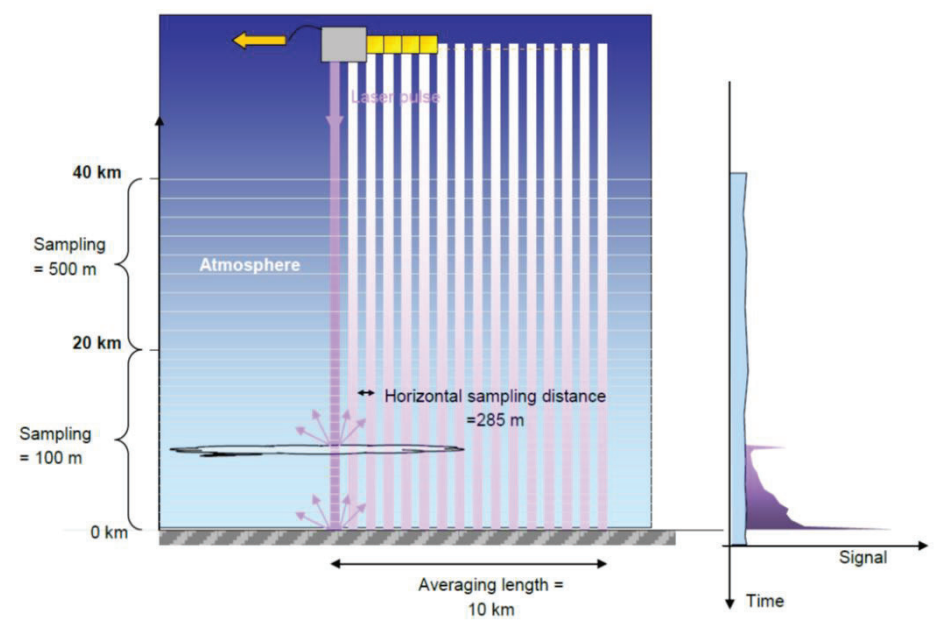

Figure 1. ATLID sampling scheme for vertical range and ground sampling 


\subsection{ATLID design description}

The ATLID instrument has a bi-static LIDAR architecture: two independent paths (for redundancy reasons) for the Transmitter Assembly (TxA) and one separated path for the Receiver Assembly (RxA). A more detailed description of all the subunits is depicted in [8][7]; Figure 2 presents the overview of the design described just below.

The TxA is based on a tripled Nd:YAG diode pumped MOPA (Master Oscillator Power Amplifier) laser, developed by Leonardo (Pomezia), and an external beam expander (EBEX), developed by Sodern (Limeil Brévannes). The UV laser emits $35 \mathrm{~mJ}$ pulses at the PRF rate of $51 \mathrm{~Hz}$ at $354.8 \mathrm{~nm}$, with timing synchronous with the detection chain.

The $\mathrm{RxA}$ is a $620 \mathrm{~mm}$ aperture $\mathrm{SiC}$ telescope with attached $\mathrm{SiC}$ focal plane assembly. The atmospheric echo is filtered from the solar background via a narrow band filter and via a blocking filter limiting the instrument field of view to equivalent $66.5 \mu \mathrm{rad}$ total cone angle. The echo is then entering the HSRE section (developed by RUAG), which extracts the cross-polarisation signal and spectrally splits the co polarized signal into two channels: the HSRE Fabry Perot etalon transmitted signal is collected by the so called Mie Channel, while the reflected signal is collected on the Rayleigh channel. The Fabry Perot function, combined with the backscattered spectrum, induces a small signal spectral cross talk between the channels that is corrected in data processing. The signal is fiber coupled and acquired on an MCCD sensor that ensures very low read out noise while keeping high quantum efficiency.

Both RxA and TxA are mounted on a common stable structure assembly that maintains good passive co-alignment performance of their two independent optical paths, while an active, co-alignment, closed control loop corrects the remaining thermo-elastic line of sight (LoS) perturbation with a $10 \mathrm{~min}$ low reaction time. The co-alignment loop commands a beam steering mechanism (BSM) inside the laser in order to co-align the TxA LoS on the RxA LoS. The control loop inputs are based on the centroiding results from a sampled beam on a co-alignment sensor (CAS) that is positioned inside the focal plane. 

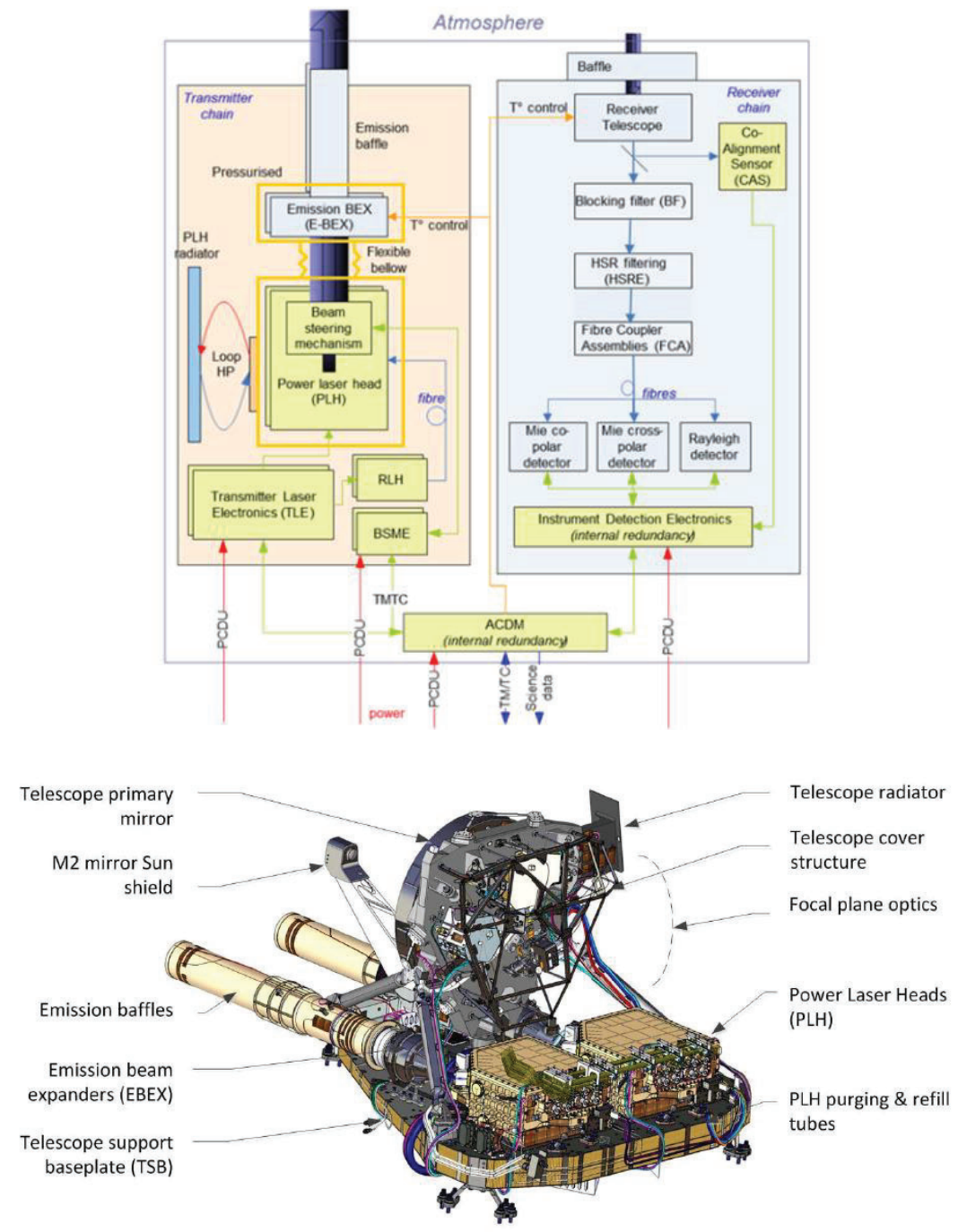

Figure 2. ATLID block diagram description and ATLID Optical Flight Model (OFM) placed on stable structure assembly

\section{ATLID PFM FINAL TEST SEQUENCE}

The early ATLID instrument integration verification steps have been described in [9][10][11]; prior to its final integration and coupling, an optical flight model and an electrical flight model have been developed and tested in parallel.

After the final coupling and closing of the instrument (see Figure 3), the qualification sequence firstly characterized the performance of the instrument in ambient clean room conditions, following which an environmental test run validated the instrument behavior within EMC, vibration and thermal vacuum (TVAC), as per Figure 4. 


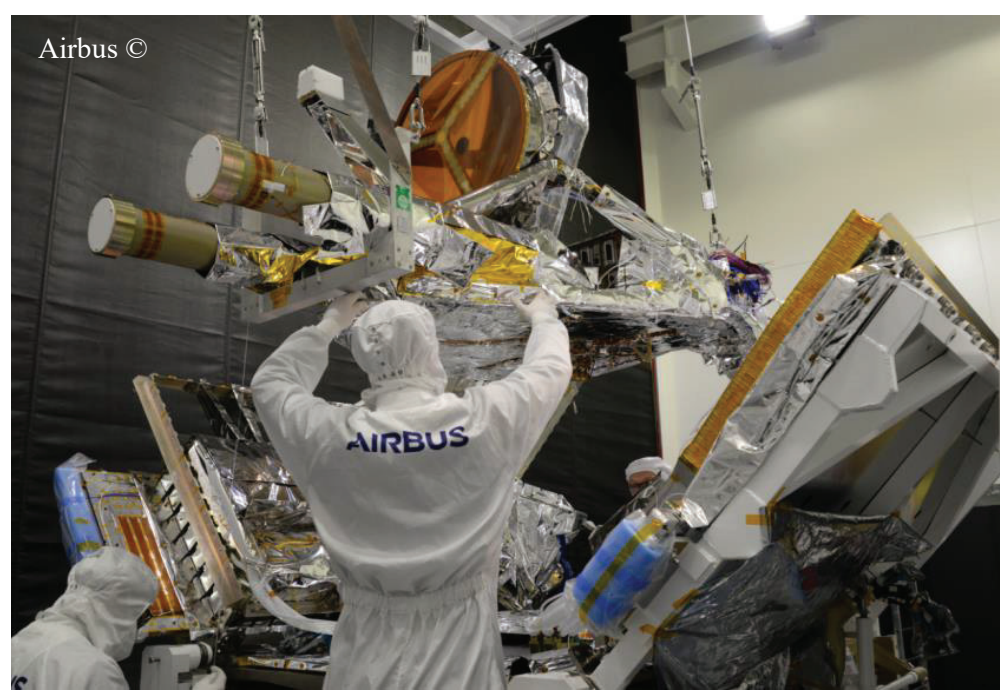

Figure 3. ATLID final coupling of OFM (top, assembly with RxA telescope and two TxA emission baffles), together with Electrical Flight Model (open structure with electronic units attached to the walls).

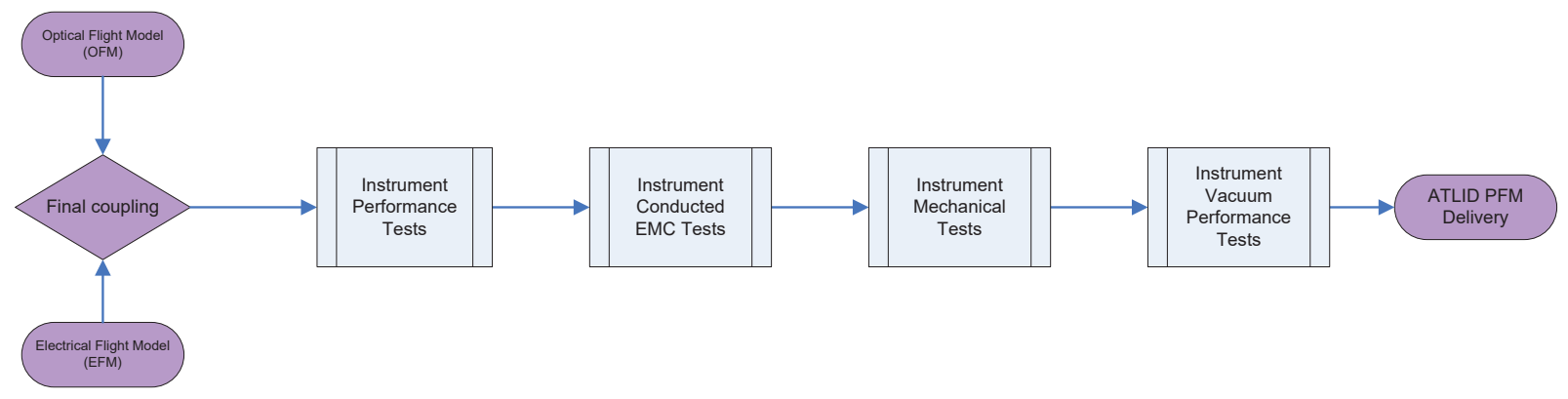

Figure 4. ATLID Proto Flight Model (PFM) test sequence steps after final coupling stage

The instrument performance at ambient is biased due to the different than flight environment thermal, vacuum and gravity conditions. But several of the performance requirements, independent from this environment, could be directly verified. Others could be at least characterized and the results extrapolated to those in vacuum.

The main parameters measured were concerning the emitter and receiver polarization, the end to end timing validation of the laser shot to detection chain acquisition synchronization, the detector over-illumination, the spectral tuning performances, the co-alignment loop validation and the stray-light.

The EMC test runs demonstrated immunity of the instrument telemetry and science data to conducted emission perturbations while in operation. 


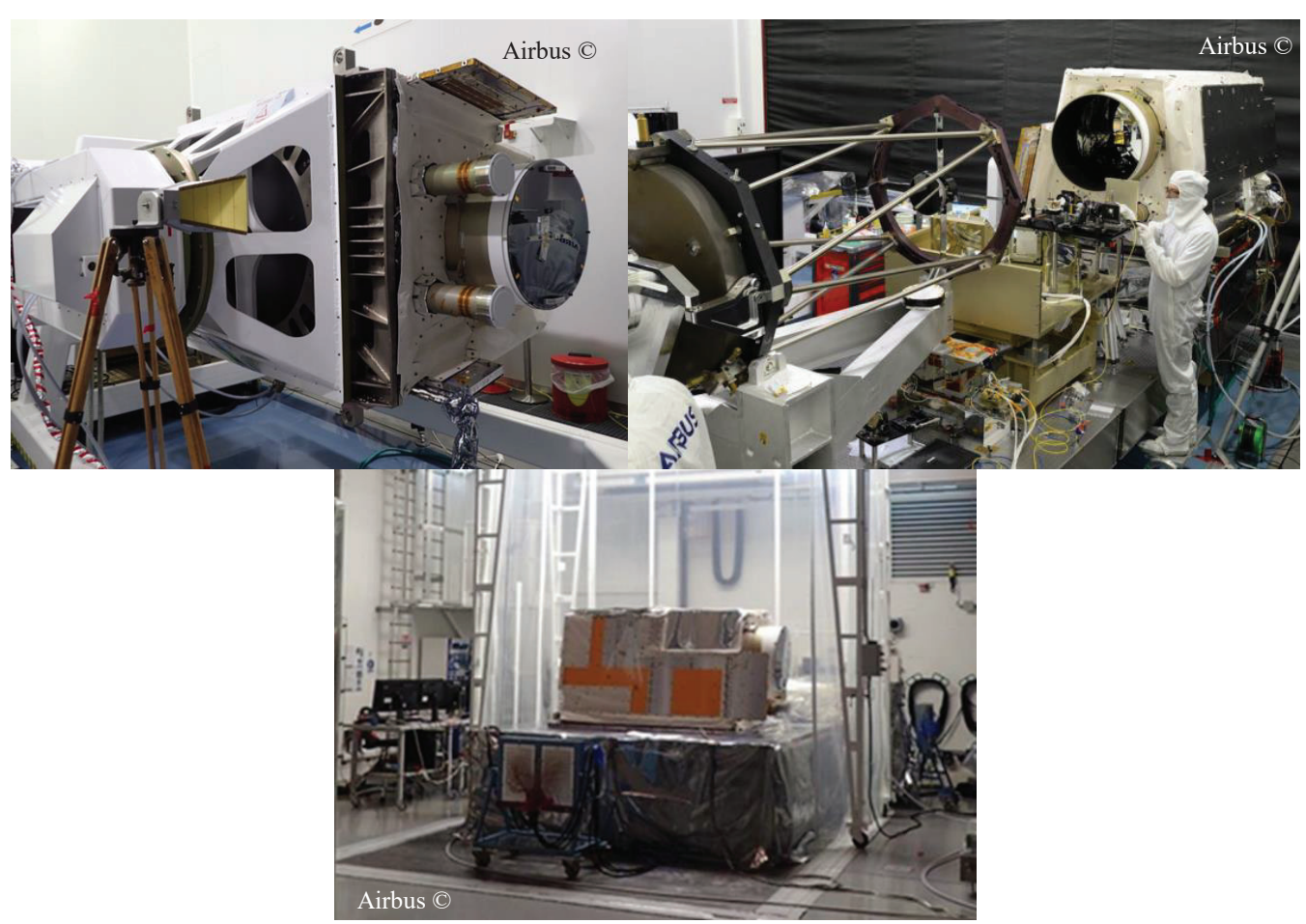

Figure 5. Top left: ATLID PFM in RE EMC test, Top right: ATLID in ambient characterization tests, Bottom: ATLID on sinus shaker

The mechanical vibration test submitted the instrument to representative flight acoustic and sinus environment. Instrument reference test before and after the vibration runs validated the instrument stability; EarthCARE is qualified against the Soyuz sinus and acoustics loads.

\section{ATLID THERMAL VACUUM TEST RUN}

The thermal vacuum test is key in the verification because it demonstrates the ability of the instrument to keep its performances in a thermal environment as close as possible to expected flight conditions and within the vacuum environment. The tests were run in Centre Spatial de Liège facility (Liège).

The thermal vacuum tests are described below in independent sections concerning the receiver part and then the transmitter part.

\subsection{Receiver characterization setup}

The instrument's high sensitivity to the simulated echo characteristics need to be dis-criminated from the possible bias or instabilities of the test setup. The simulated echo source is a tunable laser coupled to an optical system that control its divergence, line of sight, wavelength, polarization and pulse timings. Any uncontrolled variation of these parameters will induce a performance variation that will degrade the test accuracy.

The measurement setup used for the instrument characterization is shown in Figure 6. It transmits a beam of the simplified atmospheric echo:

- Generated by a frequency-tripled, Nd:YAG, narrow-band Optical Ground Support Equipment (OGSE), UV laser (source-pack outside the vacuum chamber), which is fibre-coupled;

- Through part B of OGSE FPA (OGSE multi-purpose focal plane assembly, outside the vacuum chamber, detailed in Figure 7), which allows for laser beam parameters adjustment, including polarization, line-of-sight and radiometric level, and then free path; 
- Through OGSE collimator COL70 (70 cm diameter, inside the vacuum chamber); up to ATLID telescope input.
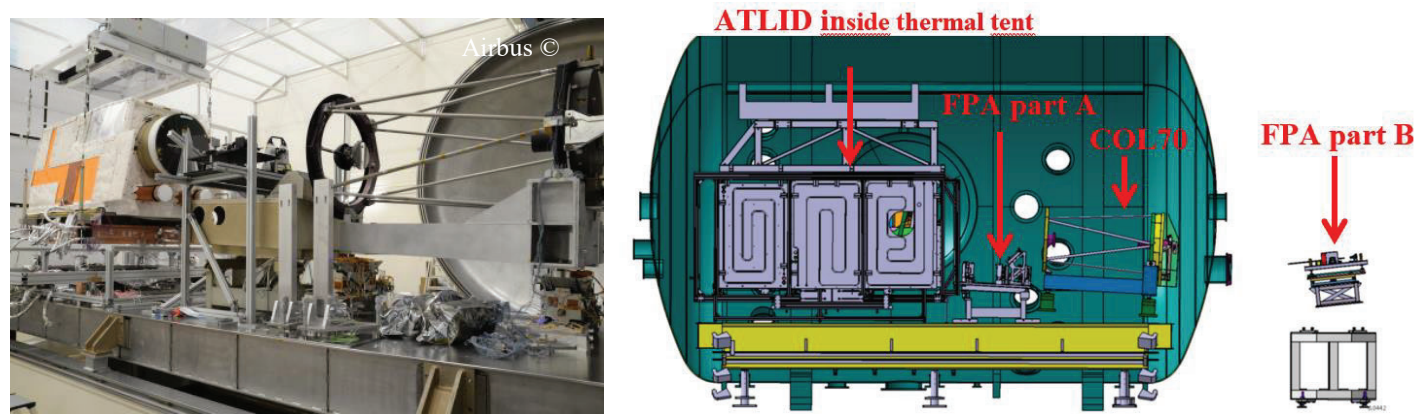

Figure 6 ATLID test configuration in vacuum chamber, with FPA (Focal Plane Assemblies) parts A\&B and collimator COL70

\subsection{Transmitter characterization setup}

The two ATLID emitters have been characterized in terms of laser pulse energy and laser beam divergence.

The additional measurement setup used for the characterization is shown in Figure 7 and indicated with FPA part A in Figure 6 (left). It can collect the radiation from both nominal and redundant lasers (yellow circles) into a single path toward COL70 inside the vacuum chamber. Laser A beam (bottom circle) is reflected on the front side of P0, while laser $\mathrm{B}$ beam (top circle) is going through prism P0 and is reflected on the back side. The wedge angle of this prism is sufficiently large to avoid any parasitic images within the FF (Far Field) image pattern $(<100 \mu \mathrm{rad})$. A camera system is placed at the output of COL70 system, outside the vacuum chamber on FPA part B, as per Figure 6
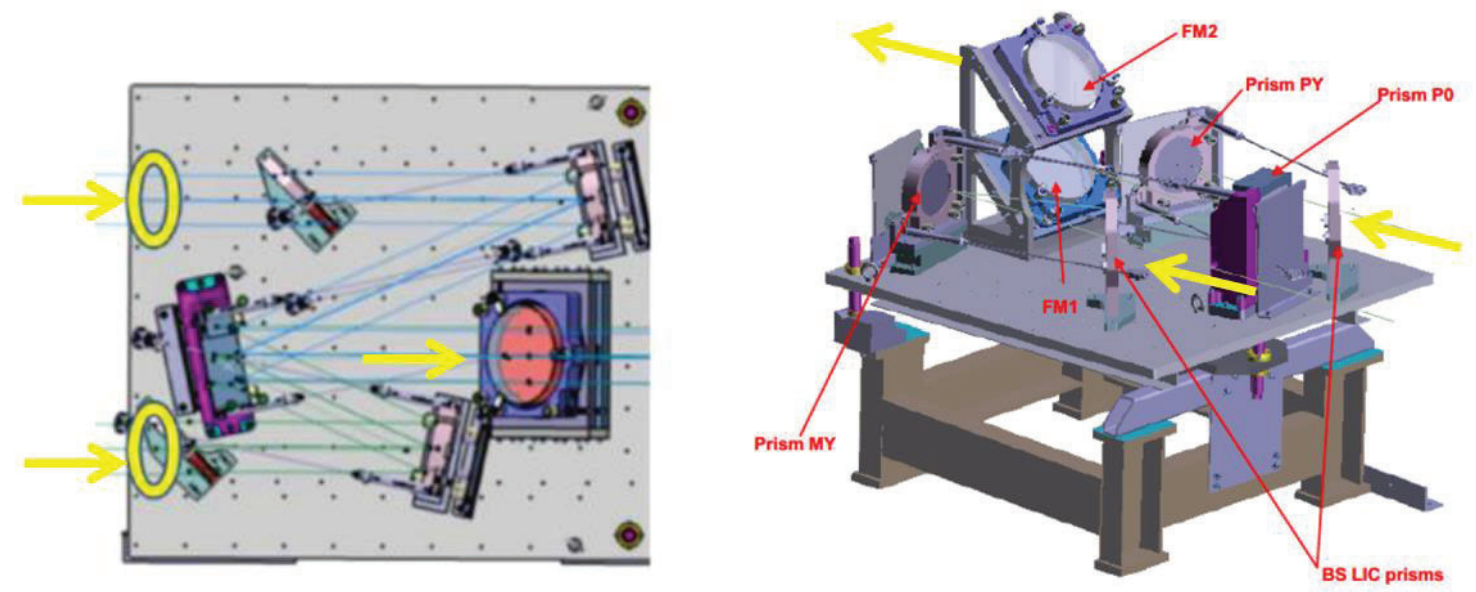

Figure 7 FPA part A, OGSE periscope setup for TxA beam collection into COL70

\subsection{Transmitter test results}

\subsubsection{Emitter pulse energy}

The objective was to estimate the energy knowledge via the internal laser photodiode monitoring. To this purpose, the absolute energy was measured before TVAC test, acquiring the entire laser beam spot. For TVAC test two additional photodiodes were installed and calibrated on the OGSE, one on its main bench (FPA part B) and one inside the vacuum chamber behind a partially reflective mirror (FPA part A). These two sensors aimed at providing a measurement reference external to the laser, all along the laser optical path, during the whole TVAC test.

An example result of the telemetry calibration is shown in Figure 8 (a) and (b) shows the overall, internal photodiode reading during the entire test, for both emitters: energies between 35 and $40 \mathrm{~mJ}$ were reached inside the power laser head. 


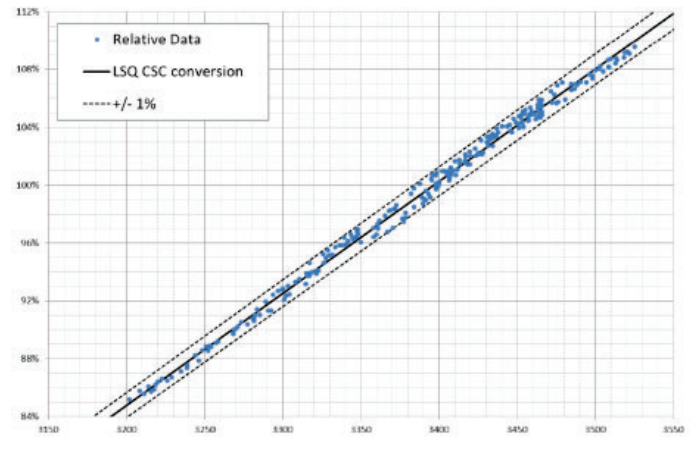

(a)

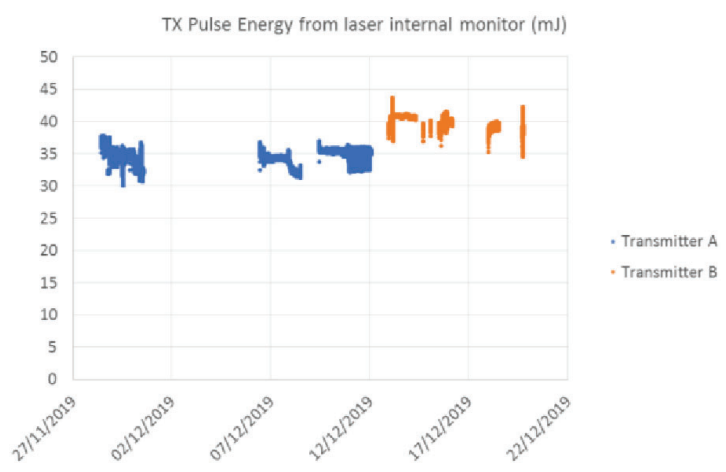

(b)

Figure 8 Example of Energy measurements performed during TVAC via the internal laser photodiode: (a) Energy telemetry calibration plotting calibrated detector measurement of emitter energy (y) against internal laser photodiode (x); (b) Overview of laser internal energy measurements during the entire vacuum phase $(\mathrm{mJ})$.

\subsubsection{Emitter divergence}

With this test a measurement of the far field pattern (FFP) of the emitted laser beams was performed, thanks to the imaging capability of the COL70 collimator OGSE. The measurements were performed in vacuum, collecting OGSE images on a shot-to-shot basis (instantaneous FFP). Post processing averaging was done to compute integration over an equivalent of $10 \mathrm{~km}$ horizontal span. Additional measurements were also done to verify the capability to thermally adjust the external beam expander (EBEX) defocus and to quantify the thermal sensitivity; in this second phase, emission defocus calibration (EDC) mode is commanded, inducing a slow scan of EBEX temperature (Figure 9). A special test procedure is used to optimize the number of steps (down to 4 or 5 steps) for this ground test, in order to reduce test duration compared to a finer stepping in flight.

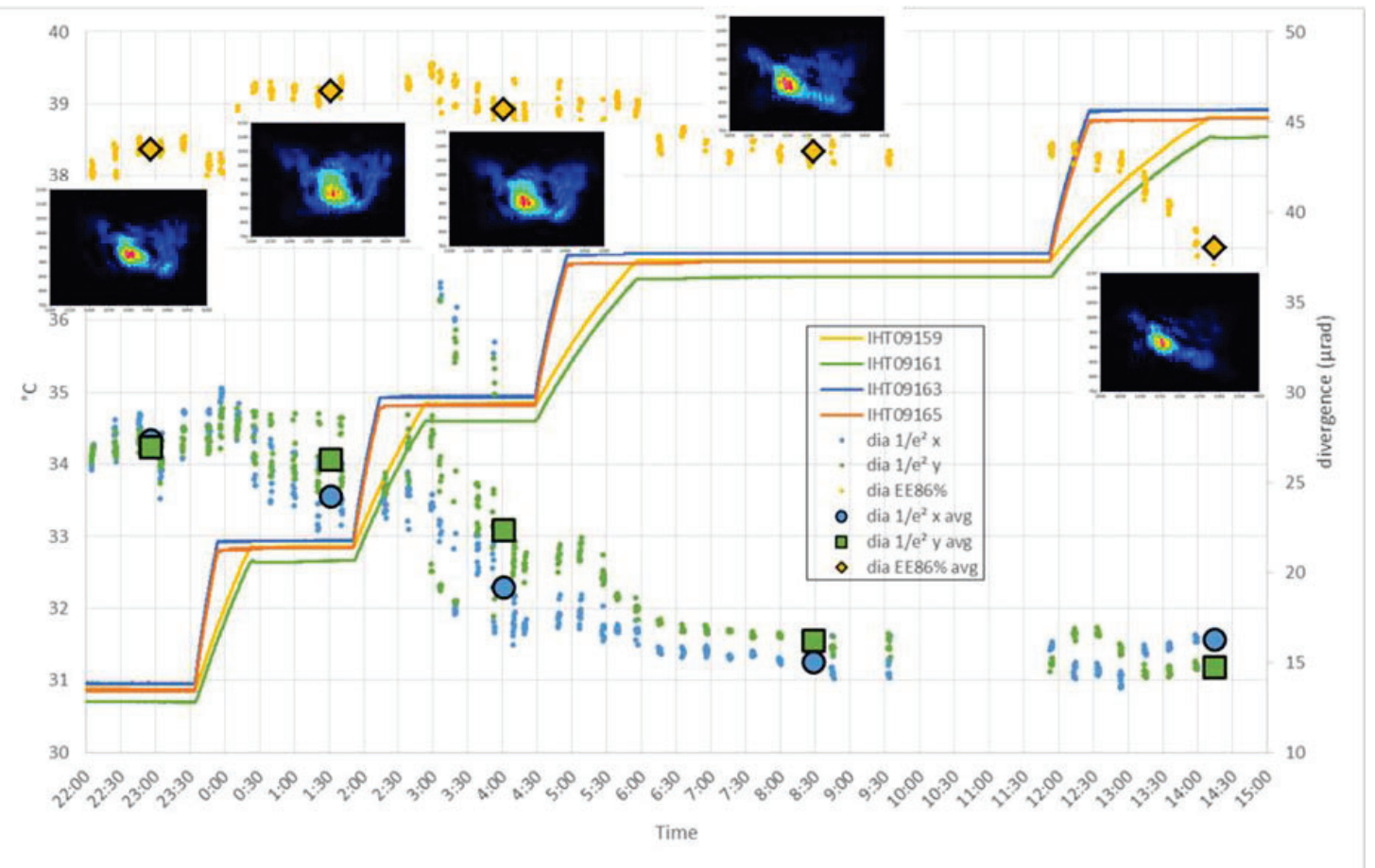

Figure 9 shows the far field measurement results during EDC; as expected, the divergence is in the 15-45 $\mu \mathrm{rad}$ range (with angular resolution lower than $2 \mu \mathrm{rad}$ and accuracy within $\pm 5 \mu \mathrm{rad}$ ). Far field pattern images are reported in the figure in correspondence to the different tests' EBEX temperatures. 
The FFP was also acquired during the simulation of orbital cycle effects, i.e., for $\mathrm{a} \pm 1{ }^{\circ} \mathrm{C}$ temperature oscillation on the transmitter thermal interface. Figure 10 shows the evolution of encircled energy divergence during about 6 cycles. The FF images corresponding to the minimum and maximum temperatures are reported inside the plot.

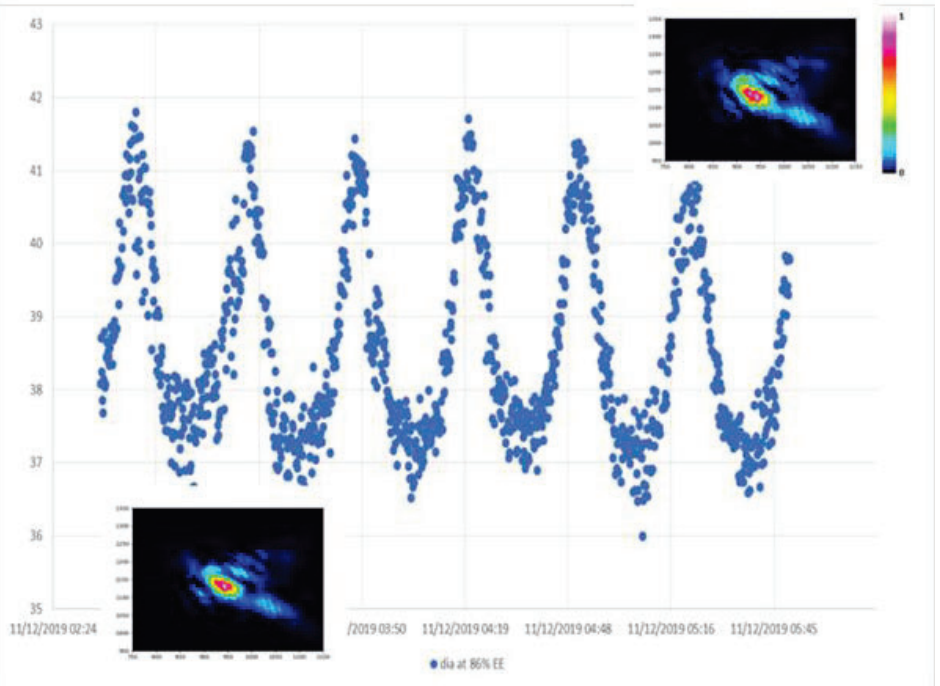

Figure 10 Far field measurement results during simulation of orbital cycles $\left( \pm 1^{\circ} \mathrm{C}\right.$ on the laser $)$; the variation of beam divergence (left scale, diameter of $86 \%$ encircled energy) plotted over time (overlaid, camera acquired far field images).

\subsection{Receiver test results}

The ATLID receiver has been characterized in terms of polarization, field of view, radiometric stability, dark current.

\subsubsection{Polarization characterization}

The objective here was to characterize the response of the ATLID receiver for each of the three channels, for both polarization axes, and over at least one free spectral range of HSRE (Figure 11). To this purpose, the OGSE emission line-of-sight is centered in instrument field-of-view, radiometric level is adjusted to be in the instrument measurement dynamic, and:

- Scans of the OGSE laser wavelength are performed, for vertical and horizontal linear polarization directions, and the channels' relative responses are extracted;

- Rotations of the OGSE beam linear polarization orientation are performed, for OGSE source-pack laser wavelength set at HSRE response peak, and the channels' relative responses are extracted. 


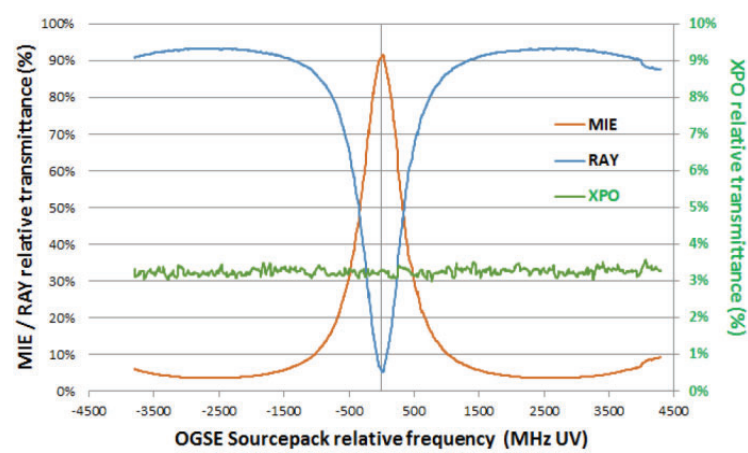

(a)

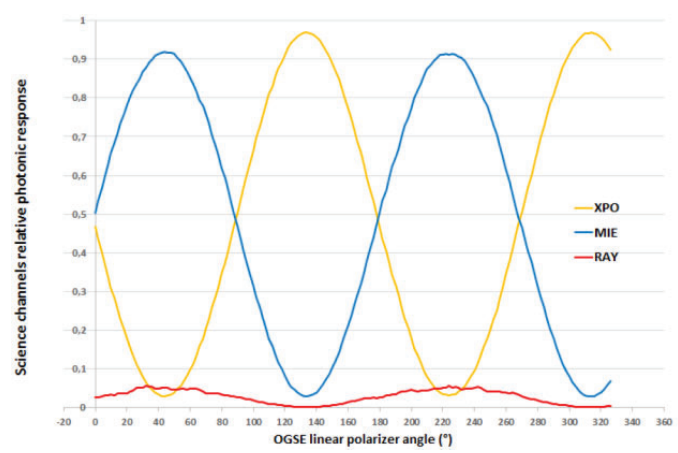

(b)

Figure 11 Example of polarization measurements performed during TVAC; channels' relative responses versus: (a) OGSE frequency scan; (b) OGSE linear polarization rotation.

\subsubsection{Field of view characterization}

The objective here was to evaluate the reception field-of-view angular diameter, in order to assess the collecting solid angle of the instrument. The same test also permits to assess the reference receiver line-of-sight with respect to the coalignment sensor. To this purpose, the OGSE emission line-of-sight is scanned around the center of the instrument fieldof-view, radiometric level is adjusted to be in the instrument measurement dynamic, and the channels' responses are extracted and normalized. The angular width of the normalized response gives the angular diameter of the instrument reception field-of-view, as can be seen in Figure 12. It is noticeable that the value in flight is predicted slightly above that measured on ground, at $66.5 \mu \mathrm{rad}$, due to gravity defocus compensation.

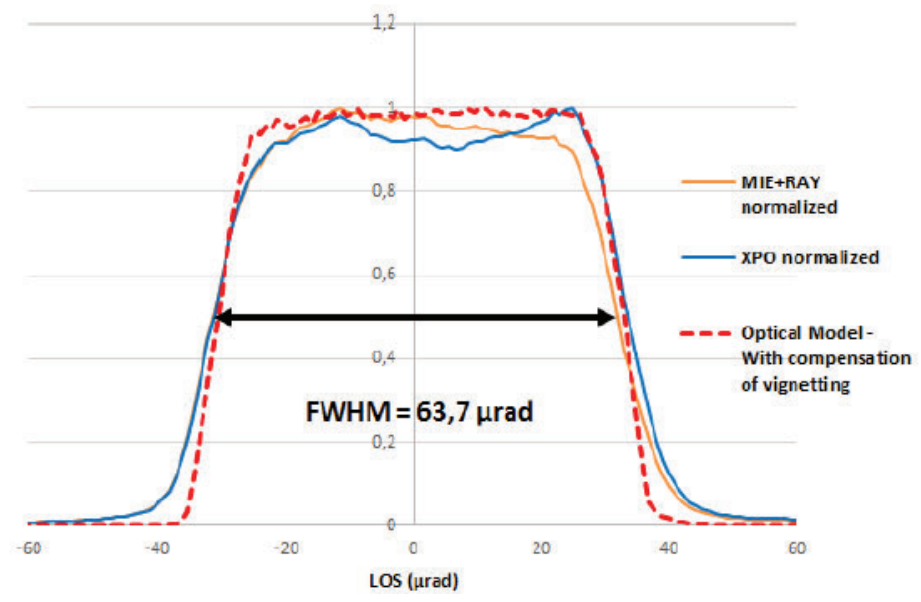

Figure 12 Example of instrument field-of-view characterization measurements performed during TVAC; channels' normalized responses vs OGSE Line-of-Sight scan.

\subsubsection{Radiometric stability characterization}

The objective was to characterize the relative instrument response variation for each of the three science channels, with varying environment. To this purpose, the OGSE emission line-of-sight is centered in the instrument field-of-view, OGSE laser wavelength is set at HSRE response peak and the thermal environment in the vacuum tank is managed in order to describe the equivalence of the foreseen orbital thermal cycle. The channels' signals are extracted and their stabilities during thermal cycle are calculated. As the OGSE frequency drift during the measurement contributes to signal 
variation during the test, its contribution is calculated and accounted for in the measured radiometric stability assessment, in order to assess the impact of thermal cycle only.

\subsubsection{Dark current characterization}

The objective was to record the dark signal map and the dark noise in operational conditions at a $-30^{\circ} \mathrm{C}$ set point for the detector CCD. To this purpose, the OGSE does not emit any signal and ATLID is in dark current calibration (DCC) mode without laser emission. The output of this test allows filling the calibration database to be provided with the instrument and to be used by the EarthCARE ground processer. The dark noise all along the atmospheric profiles is below the defined $2.2 \mathrm{e}$ - rms success criteria for all channels, validating the ATLID performance model budgeted value.

\section{ATLID PERFORMANCE TEST RESULTS}

\subsection{Performance analysis}

The performance prediction is made using the test results obtained with an artificial, atmospheric echo simulation to reconstruct the instrument performance; as shown above during the instrument testing, no direct atmospheric test is performed. In particular, the test source spectrum is a monochromatic, monomode, longitudinal laser line at $355 \mathrm{~nm}$, while in flight the received backscattered signal spectrum will be broadened by Rayleigh backscattering superimposed onto a variable Mie spectrum line (Figure 13).
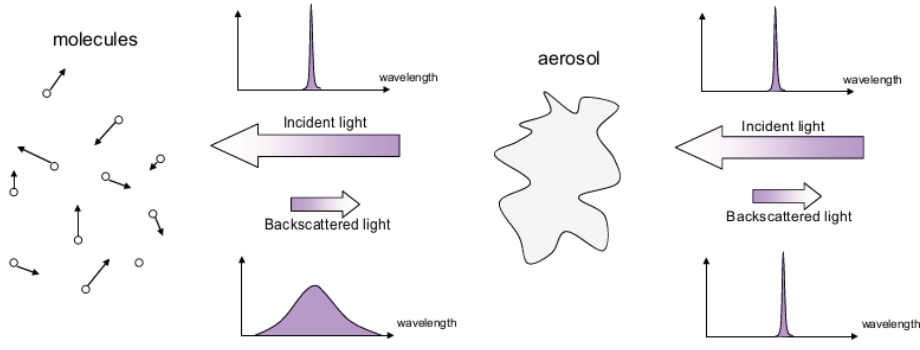

Figure 13 Illustration of the backscattered spectrum.

Also, the echo will be partially depolarized by the atmospheric scene; for example, Sahara aerosols can depolarize up to $30 \%$ of the polarized light.

The instrument performance model is updated based on the instrument test results shown above. In particular, the receiver channel cross talks are verified and calibrated. This concerns at first the polarization cross talk characteristic between co polar and cross polar channels that is checked via input beam of pure Co polarization, or pure Cross polarization. The spectral cross talk is also confirmed, by verifying the instrument spectrum response of the HSRE. The cross talk correction needs are shown in Figure 14.

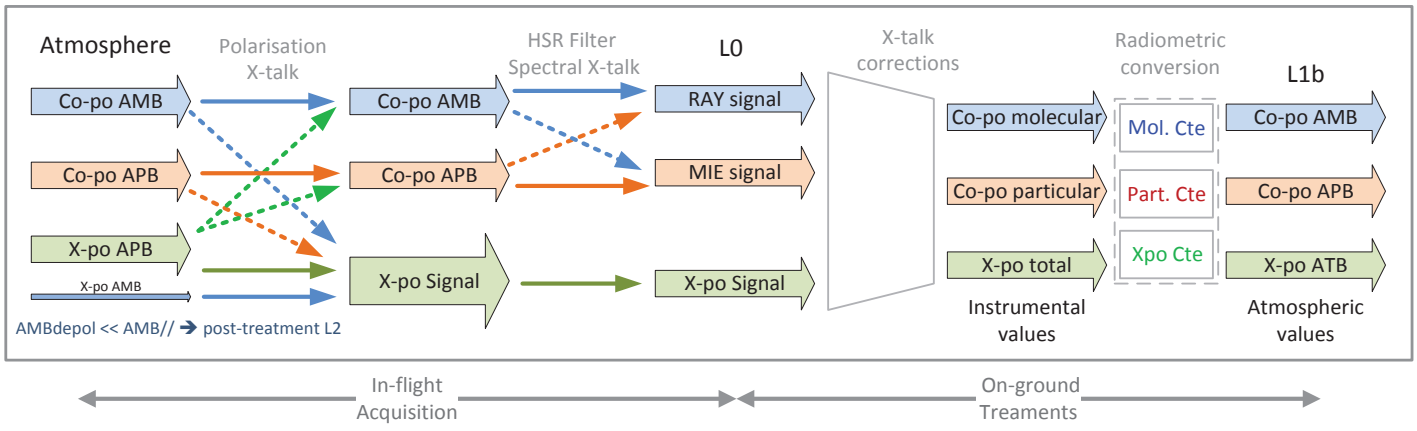

Figure 14 Illustration of signal cross talks and need for cross talk corrections. 


\subsection{Performance summary tables}

Table 1 shows the ATLID instrument radiometric characteristics predicted for flight and Table 2 shows the ATLID detection performance.

Table 3 shows the derived ATLID LIDAR performance of backscatter absolute retrieval accuracy, based on the relative retrieval accuracy on each channel and the absolute calibration of the LIDAR constant. The accuracy is given at $10 \mathrm{~km}$ altitude sample, on a $10 \mathrm{~km}$ horizontal integration length, for three types of scenes:

- a sub visible cirrus: faint cirrus at the limit of instrument detection, $\beta=8 \times 10^{-7} \mathrm{sr}^{-1} \mathrm{~m}^{-1}$

- $\quad$ a thin cirrus : $\beta=1.4 \times 10^{-5} \mathrm{sr}^{-1} \mathrm{~m}^{-1}$

- a depolarization cirrus : cirrus with $10 \%$ depolarization ratio, $\beta_{\mathrm{par}}=2.6 \times 10^{-5} \mathrm{sr}^{-1} \mathrm{~m}^{-1}$ and $\beta_{\mathrm{per}}=2.6 \times 10^{-6} \mathrm{sr}^{-1}$ $\mathrm{m}^{-1}$

Table 1. ATLID radiometric characteristics prediction for flight

${ }^{1}$ value predicted in flight is different from ground measured value due to gravity focusing effects;

${ }^{2}$ End of life value without etalon transmission;

${ }^{3}$ photo flux ratio characteristics of HSRE.

\begin{tabular}{|c|c|c|}
\hline Item & Value & Units \\
\hline Receiver field of view & $66.5^{1}$ & $\mu \mathrm{rad}$ \\
\hline Telescope diameter & 620 & $\mathrm{~mm}$ \\
\hline Solar background filter equivalent bandwidth & 710 & $\mathrm{pm}$ \\
\hline Mie Co Polar transmission $\mathrm{EOL}^{2}$ & 45 & $\%$ \\
\hline Mie Cross Polar transmission $\mathrm{EOL}^{2}$ & 43 & $\%$ \\
\hline Rayleigh transmission $\mathrm{EOL}^{2}$ & 43 & $\%$ \\
\hline (molecular to molecular channel) $\mathrm{C}_{-} \mathrm{mm}^{3}$ & 75 & $\%$ \\
\hline (molecular to particle channel) $\mathrm{C}_{-} \mathrm{mp}^{3}$ & 25 & $\%$ \\
\hline (particular to molecular channel) C_pm ${ }^{3}$ & 16 & $\%$ \\
\hline (particular to particular channel) C_pp ${ }^{3}$ & 84 & $\%$ \\
\hline Laser pulse energy & 35 & $\mathrm{~mJ}$ \\
\hline Pulse repetition frequency & 51 & $\mathrm{~Hz}$ \\
\hline Transmitter field of view & 36 & $\mu \mathrm{rad}$ \\
\hline Transmitter EOL transmission & 89 & $\%$ \\
\hline polarisation & Linear & - \\
\hline
\end{tabular}


Table 2. ATLID radiometric characteristics prediction for flight

${ }^{1}$ (MieCoPo / MieCross / Rayleigh);

2 from $10 \mathrm{e}$ - to the top; ${ }^{3}$ from $1 \mathrm{e}-$ to $10 \mathrm{e}-$.

\begin{tabular}{|r|c|c|}
\hline Detection performance & Value & Units \\
\hline Vertical resolution from 0 to $20 \mathrm{~km}$ & 100 & $\mathrm{~m}$ \\
\hline Vertical resolution from 20km to 40km & 500 & $\mathrm{~m}$ \\
\hline Vertical cross talk & $\begin{array}{c}4.5 \% \text { on } 500 \mathrm{~m}, \\
11 \% \text { on } 100 \mathrm{~m}\end{array}$ & \\
\hline Computed quantum efficiency & $79 / 75 / 79^{1}$ & $\%$ \\
\hline Linearity & $\begin{array}{r}<+/-1^{2} \\
<+/-2^{3}\end{array}$ & $\%$ \\
\hline Noise worst case & 2.2 & $\mathrm{e}-\mathrm{rms}$ \\
\hline Dynamic margin on channel vs predicted worst signal & $18 / 5 / 12^{1}$ & $\%$ \\
\hline
\end{tabular}

Table 3. ATLID LIDAR retrieval accuracy

\begin{tabular}{|c|c|c|c|}
\hline $\begin{array}{r}\text { LIDAR absolute backscatter retrieval accuracy } \\
10 \mathrm{~km} \text { horizontal integration }\end{array}$ & Typical BOL & $\begin{array}{l}\text { Worst Case } \\
\text { EOL }\end{array}$ & unit \\
\hline Mie Co Polar on sub visible cirrus : $\beta=8 \times 10^{-7} \mathrm{sr}^{-1} \mathrm{~m}^{-1}$ & 31 & 48 & $\%$ \\
\hline Mie Co Polar on thin cirrus : $\beta=1.4 \times 10^{-5} \mathrm{sr}^{-1} \mathrm{~m}^{-1}$ & 6 & 8 & $\%$ \\
\hline Mie Cross polar on depol. cirrus : $\beta=2.6 \times 10^{-6} \mathrm{sr}^{-1} \mathrm{~m}^{-1}$ & 19 & 23 & $\%$ \\
\hline Rayleigh (above cirrus 10km) & 12 & 17 & $\%$ \\
\hline
\end{tabular}

\section{ATLID NEXT STEPS}

\subsection{Integration in the satellite and follow up}

The ATLID instrument verification tests are completed and the performances have been updated as described in above section. In March 2020, final mechanical integration has been completed and the ATLID alignment on the satellite platform is confirmed in the good range (of $<0.42 \mathrm{mrad}$ around satellite Nadir pointing). This will allow achieving full compliance to the EarthCARE inter instruments alignment performance requirements of ATLID with regards to MSI, $\mathrm{BBR}$ and CPR.

The electrical and functional coupling with the platform confirmed the good operability of the instrument in the hands of EarthCARE prime operator.

The instrument has been controlled at ambient before and after integration showing no deviation on its main performance (LoS, detection chain, laser energy, relative channel transmission). The same performance checks will be repeated till launch campaign to confirm the instrument health; in particular, the satellite will undergo functional tests, plus the satellite level vibration and thermal vacuum test where the ATLID instrument will be operated. EarthCARE launch is expected in first half of 2023

\subsection{Calibration and in orbit validation plan}

The ATLID on ground test allowed calibrating and characterizing the instrument as depicted in section 4.1. Once in orbit, dedicated processing is planned to ensure calibration maintenance of the LIDAR product.

In flight, a continuous monitoring of instrument response will be performed on stratospheric backscatter, assumed to contain pure Rayleigh signal, and on cloud or ground echoes, assumed to contain pure Mie signal as per Figure 15. With such data, the channel cross talk can be calibrated as well as the LIDAR constant based on well-known models of Rayleigh pure backscattering. Those activities do not require LIDAR acquisition interruption, only post-processing of identified scenes.

On the contrary, regular checks of the HSRE tuning and the detector dark current of the vertical profile pixels will be performed in a dedicated operation mode. Fine spectral tuning calibration mode, with emitter wavelength sweep, will control HSRE peak response; dark current calibration mode, with no laser emission, will map the vertical profile pixels dark current while the satellite is in eclipse. The duration and frequency of these checks, expected less than half an hour 
and typically per month for the dark current verification and per week for the spectral calibration, will be confirmed after in orbit commissioning activities.

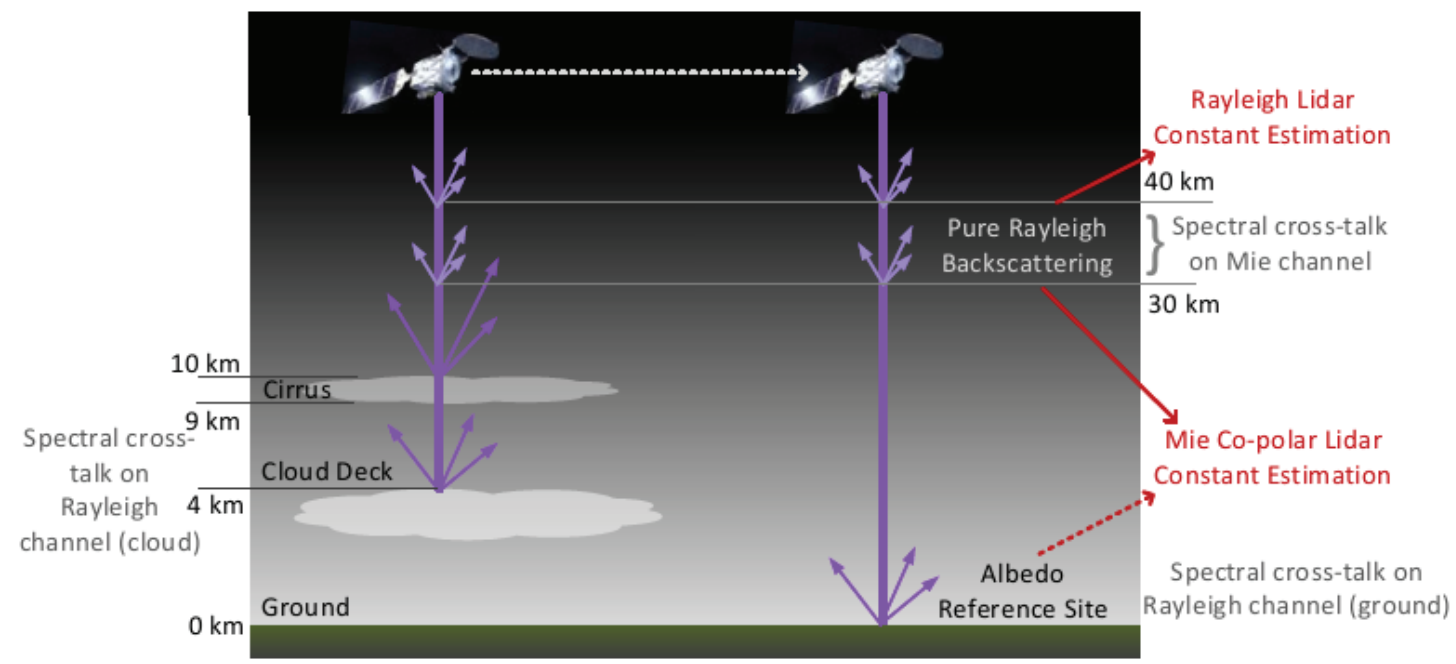

Figure 15 In flight calibration scene of the Mie and Rayleigh LIDAR constant and their channel cross talk.

\section{CONCLUSION AND PERSPECTIVES}

\subsection{Lessons learnt}

ATLID development indicates the feasibility of a UV vertical range resolved LIDAR instrument, with high spectral resolution receiver. Together with past experience on ALADIN, the authors have gained lessons learnt on the development and design criticalities.

Laser optics robustness to high fluence beam and to induced contamination has been mitigated using past experience [15][17]. The reduction of the number of high fluence exposed surfaces, the high care for contaminant removal and the intense bake out process are key elements. Optics procurement with LIDT (Laser Induced Damage Threshold) qualification batch control is mandatory, as well as LIC test at material level or at optical subsystem level.

Beyond the material and the optics parts selection and validation, the laser lifetime is depending on the pressurization sealing quality and verification, on the pumping diode lifetime, on the long term thermomechanical stability and on the possible performance compensation with the laser adjustment operation. Pressurization design at 1.2bar has been qualified on structural and thermal model; leakage has been verified on sealed housing at several steps on the integration, with a value close to the permeation limit of the sealing Viton $\mathrm{O}$ ring material. The diode lifetime has been tested over 1 year operation and has shown no degradation of their optical power within the accuracy of $+/-0.5 \%$.

The latest test running outside ATLID instrument is now the PLH flight spare life test, to be run for at least 4 months, to reveal the overall performance stability on longer term, while the ATLID mission lifetime is 36months.

Among important ALADIN lessons learnt are the so called "hot pixels" observed on that instrument's ACCD. Their experience is for a small but regularly increasing number of pixels that have shown dark current anomalies over time, with unexpected dark current increase, leading to science data systematic error. A new calibration method was elaborated to refresh the dark current map 4 times a day, whilst minimizing the availability impact, but correcting the error [16]. ATLID's similar MCCD technology has been evaluated against the ALADIN observation and is considered less at risk due to the 10 times lower number of signal accumulations, limiting the dark current error effect, and due to the different charge transfer sequence. As precaution, ATLID will characterize its dark current trend in particular in next satellite TVAC test with nominal $-30^{\circ} \mathrm{C}$ temperature at detector level. Alternatives have already been defined for more frequent calibration method on-orbit, in case of need. 
Also, the ATLID campaign has shown polarization control for reaching the $1 \%$ accuracy domain to be delicate and require precaution on verification setup. More globally the complexity of the verification is linked to the instrument being a radiometric instrument extremely sensitive in beam spatial characteristics, in optical frequency, polarization and pulse timing. The GSE source is required to be stable simultaneously in this entire domain: low field of view below $65 \mu \mathrm{rad}$, highly selective wavelength resolution at $100 \mathrm{MHz}$ level, $<1 \%$ polarization purity and acquisition temporal window synchronization of typically less than $1 \mu$ s.

\subsection{Conclusion}

Since the completion of the full instrument assembly in 2019 [9], ATLID has been subjected to an ambient performance test campaign [10][11], followed by a successful environmental qualification test campaign, including performance calibration and characterization in thermal vacuum conditions. ATLID has been successfully operated as a self-standing instrument and all performance aspects, including transmitter and receiver characteristics, have been found to be in line with targeted expectations and compliant with all major science, in-flight, end of life, performance requirements. This includes under worst case scenarios, where is assumed instrument performance degradation as well as operation in less favorable orbital points regarding sun illumination conditions. The instrument has been delivered to the EarthCARE industrial Prime for assembly and integration onto EarthCARE satellite platform, a task that was completed in May 2020. Instrument and platform interface verifications are now approaching an end, with the execution of the last performance checks and functional testing at system level recently completed. With the completion of the foreseen platform qualification test campaign and flight acceptance review in 2022, the launch of EarthCARE satellite in 2023, and the first 6 months of in-orbit commissioning phase, ATLID will be ready to start its 3 year long mission, to measure aerosols and thin clouds from space. Data from ATLID is expected to provide a major contribution to the earth observation science community's understanding of the cloud-aerosol-radiation interaction and Earth radiative balance, and in development of more reliable climate and numerical weather prediction models.

\section{REFERENCES}

\section{References}

[1] EarthCARE-Clouds, Aerosols and Radiation Explorer. Available online: http://esamultimedia.esa.int/docs/SP_1279_1_EarthCARE.pdf (accessed on 5.1.2021).

[2] Lefebvre, A.; Hélière, A.; Albiñana, A.P.; Wallace, K.; Maeusli, D.; Lemanczyk, J.; Lusteau, C.; Nakatsuka, H.; Tomita, E. EarthCARE mission, overview, implementation approach and development status. In Earth Observing Missions and Sensors: Development, Implementation, and Characterization IV; International Society for Optics and Photonics: Bellingham, WA, USA, 2016; Volume 9881.

[3] Hélière, A.; Wallace, K.; Carmo, J.P.D.; Lefebvre, A. Earth Cloud, Aerosol and Radiation Explorer optical payload development status. In Sensors, Systems, and Next-Generation Satellites XXI; International Society for Optics and Photonics: Bellingham, WA, USA, 2017; Volume 10423.

[4] Lefebvre, A. EarthCARE mission: Approaching launch. In Proceedings of the IEEE International Geoscience and Remote Sensing Symposium (IGARSS) 2018, Valencia, Spain, 23-27 July 2018.

[5] Wehr, T.; Eisinger, M.; Koopman, R.; Lefebvre, A.; Maeusli, D.; Wallace, K.; Pereira Do Carmo, J.; Livschitz, J.; Maksym, S. Status of ESA's EarthCARE Mission Preparation. In Proceedings of the IEEE International Geoscience and Remote Sensing Symposium (IGARSS) 2020, Waikoloa, HI, USA, 19-24 July 2020.

[6] Illingworth, A.J.; Barker, H.W.; Beljaars, A.; Ceccaldi, M.; Chepfer, H.; Clerbaux, N.; Cole, J.; Delanoë, J.; Domenech, C.; Donovan, D.P.; et al. The EarthCARE Satellite: The Next Step Forward in Global Measurements of Clouds, Aerosols, Precipitation, and Radiation. Bull. Am. Meteorol. Soc. 2015, 1311-1322, doi:10.1175/BAMS-D-12-00227.1.

[7] Hélière, A.; Wallace, K.; Carmo, J.P.D.; Lefebvre, A.; Eisinger, M.; Wehr, T. A Development status of the EarthCARE Mission and its atmospheric Lidar. In Earth Observing Systems XXI; International Society for Optics and Photonics: Bellingham, WA, USA, 2016; Volume 9972. 
[8] Carmo, J.P.D.; Chassat, F.; Hélière, A.; Toulemont, Y.; Lefevre, A. ATLID, ESA atmospheric lidar: Manufacture and test results of instrument units. In Proceedings of the International Conference on Space Optics (ICSO) 2016, Biarritz, France, 18-21 October 2016.

[9] De Villele, G.; Carmo, J.P.D.; Helière, A.; Lefebvre, A.; Barbaro, L.; Belhadj, T.; Chassat, F.; Corselle, B.; Evin, R.; Feral, M.; et al. ATLID, ESA Atmospheric LIDAR: Integration of instrument and tests. In Proceedings of the International Conference on Space Optics (ICSO) 2018, Chania, Greece, 9-12 October 2018.

[10] Carmo, J.P.D.; De Villele, G.; Helière, A.; Wallace, K.; Lefebvre, A.; Chassat, F. ATLID, ESA Atmospheric backscatter LIDAR for the ESA EarthCARE Mission. Counc. Eur. Aerosp. Soc. CEAS Space J. 2019, 11, 423435.

[11] Pereira do Carmo, J.; Wallace, K.; Lefebvre, A. Flight LIDAR development and qualification for the ESA Earth Cloud Aerosol and radiation explorer (EarthCARE) mission. In Proceedings of the IEEE International Geoscience and Remote Sensing Symposium (IGARSS) 2020, Waikoloa, HI, USA, 19-24 July 2020.

[12] Wallace, K.; Hélière, A.; Lefebvre, A.; Eisinger, M.; Wehr, T. Status of ESA's EarthCARE mission, passive instruments payload, In Earth Observing Systems XXI; International Society for Optics and Photonics: Bellingham, WA, USA, 2016; Volume 9972.

[13] Tomiyama, N.; Tomita, E.; Furukawa, K.; Nakatsuka, H.; Seki, Y.; Aida, Y.; Okada, K.; Maruyama, K.; Ishii, Y.; Ohno, Y.; et al. EarthCARE/CPR development status and performance. In Remote Sesning of Clouds and Atmosphere XXV; International Society for Optics and Photonics: Bellingham, WA, USA, 2020; Volume 11531.

[14]Domenech, C.; Donovan, D.P.; Barker, H.W.; Bouvet, M.; Lopez-Baeza, E. Improvement of broadband radiance to flux conversion by using the synergy between active and passive remote sensing instruments. In SPIE Remote Sensing, Florence, Italy, International Society for Optics and Photonics: Bellingham, WA, USA, 2007; Volume 6745.

[15] Straume, A.G.; Rennie, M.; Isaksen, L.; de Kloe, J.; Marseille, G.J.; Stoffelen, A.; Flament, T.; Stieglitz, H.; Dabas, A.; Huber, D.; et al. ESA's space-based Doppler wind lidar mission Aeolus-First wind and aerosol product assessment results. In Proceedings of the EPJ Web Conference, Moscow, Russia, 16-20 November 2020; 2019237, 01007, doi:10.1051/epjconf/202023701007.

[16] Weiler, F.; Kanitz, T.; Wernham, D.; Rennie, M.; Huber, D.; Schillinger, M.; Saint-Pe, O.; Bell, R.; Parrinello, T.; Reitebuch, O. Characterisation of dark current measurements of the ACCDs used on-board Aeolus satellite. Atmos. Meas. Tech. Discuss. 2020, doi:10.5194/amt-2020-458.

[17]Riede, Wolfgang \& Wernham, Denny \& Schröder, Helmut \& Allenspacher, Paul \& Bartels, Nils. (2019). Qualification of laser optics for challenging space LIDAR missions. CM3C.1. 10.1364/LAC.2019.CM3C.1. 